\title{
The Data Acquisition System for the KOTO Detector
}

\section{S. Su ${ }^{* 1}$, J. Xu ${ }^{1}$, M. Campbell ${ }^{1}$, M. Tecchio ${ }^{1}$, J. Ameel ${ }^{1}$, J. Micallef ${ }^{1}$, N. Whallon ${ }^{1}$,

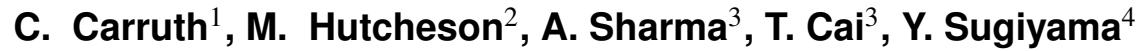

${ }^{1}$ University of Michigan

${ }^{2}$ Agnes Scott College

${ }^{3}$ University of Chicago

${ }^{4}$ Osaka University

E-mail:stephsu@umich.edu

The goal of KOTO experiment at J-PARC is to discover and measure the rate of the rare decay $K_{L} \rightarrow \pi^{0} v \bar{v}$, for which the Standard Model predicts a branching ratio of $(2.4 \pm 0.4) \times 10^{-11}$. The experiment is a follow-up to E391 at KEK with a completely new readout electronics, trigger and data acquisition system. The KOTO DAQ comprises a front-end 14-Bit, $125 \mathrm{MHz}$ ADC board, a two-level hardware trigger electronics, and a third level software trigger. The ADC board processes the detector signals through a low pass filter before digitization. The digitized pulses are stored inside a $4 \mu s$ deep pipeline while waiting for the first level trigger decision which is based on a minimum energy deposition in the CsI calorimeter in anti-coincidence with signals in veto detectors. Data is then buffered inside a second level trigger board, which calculates the centerof-energy of the event. Data accepted by the second level trigger board is read out via a front panel $1 \mathrm{~Gb}$ Ethernet port into a computer cluster through a ethernet switch using user datagram protocol. After several commissioning runs in 2011 and 2012, KOTO has taken the first physics run in May 2013. In this paper, we will review the performance of the DAQ during this run as well as plans to upgrade the clock distribution system and the overall trigger hardware connectivity. Finally we present a redesign of the Level 2 and Level 3 triggers and readout electronics which will be able to accommodate the increase in data rate expected in the next few years.

Technology and Instrumentation in Particle Physics 2014

2-6 June, 2014

Amsterdam, the Netherlands

\footnotetext{
* Speaker.
} 


\section{Introduction to The KOTO Experiment}

The universe is composed of matter and antimatter. Due to the symmetry breaking from the standard model, we ought to observe equal amount of matter as well as antimatter. However, there is a dominance of matter over antimatter in our universe and $\mathrm{CP}$ violation is required to explain this phenomenon. One famous example of CP violation is the $K_{L}^{0} \rightarrow \pi^{+} \pi^{-}$decay; another example, and the focus of this experiment is, $K_{L}^{0} \rightarrow \pi^{0} v \bar{v}$.

The KOTO experiment is located at Japan Proton Accelerator Research Complex (J-PARC) at Tokai, Japan. It studies the decay mode of $K_{L} \rightarrow \pi^{0} v \bar{v}$. This particular rare decay has a branching ratio of $(2.4 \pm 0.4) \times 10^{-11}[1]$ and it undergoes second-order flavor-changing neutral current (FCNC), whereas the first-order FCNC is prohibited by the Standard Model. The goal of the experiment is to measure the branching ratio of $K_{L} \rightarrow \pi^{0} v \bar{v}$ by reaching the Standard Model branching ratio sensitivity as well as probing for new physics. Previous direct limits on the $K_{L}$ branching ratio have been measured by experiment E391a at KEK, the best being $2.6 \times 10^{-8}$ [2] at $90 \%$ confidence level (CL). An indirect limit of $1.7 \times 10^{-9}$ is derived from experiment E949 at BNL[3] through the Grossman-Nir relation[4]. Section 2 and 3 will cover the KOTO experimental setup, and the current data acquisition (DAQ) system. Section 4 will report on a study of temperature dependence for the analog-to-digital converter (ADC) pedestal value. Finally, section 5 will report on future upgrades for the KOTO DAQ system.

\section{The Experiment Setup}

In J-PARC, the proton beam is accelerated via a $3 \mathrm{GeV}$ synchrotron and further boosted by a second synchrotron to an energy of $30 \mathrm{GeV}$. The accelerated proton beams generate $K_{L}$ mesons by colliding onto a gold target as illustrated in Figure 1. The $K_{L} \rightarrow \pi^{0} v \bar{v}$ decay can be identified by detecting the two photons from the $\pi^{0}$ decay in the final state. The cesium iodide calorimeter (CsI) is used to measure the energy and the position of the photons; other detectors surrounding CsI serve the purpose of excluding events with photons outside the fiducial region of the calorimeter and identify charged particles. The most recent run in May, 2013 used $24 \mathrm{~kW}$ proton beam power, with 2 s slow extraction spills every 6 s, which corresponds to a $K_{L}$ flux of $6.3 \times 10^{6} K_{L}$ /spill[5]. In order to reach the Standard Model sensitivity, beam intensity up to $300 \mathrm{~kW}$ is needed[6].

\section{The Current DAQ System}

Figure 2 shows a diagram of the data acquisition system[7] for the KOTO experiment. It consists of three levels - two hardware triggers and one software trigger. Analog photomultiplier (PMT) signals are digitized by the flash ADC and transmitted to the level 1 trigger (Lv1) for the energy sum calculation. The ADCs then transmit the events accepted at Lv1 to the Lv2 trigger system where the center of energy radius is calculated. Events accepted at Lv2 are sent to the Lv3 trigger where event building and data storage take place.

A single ADC board design is used to receive PMT signals from approximately $3000 \mathrm{CsI}$ calorimeter channels and 1000 veto detector channels. Each ADC module contains 16 channels. The requirements for the data acquisition system are 14-bit dynamic range for the energy measurement and one nanosecond timing resolution. To meet these requirements, the analog PMT signals 


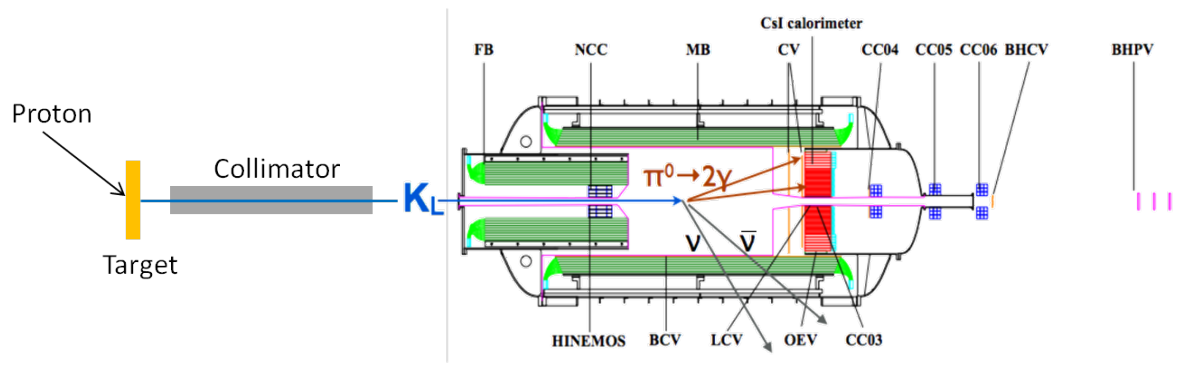

Figure 1: A schematic of the KOTO Experiment. $K_{L}$ mesons are generated from the collision of protons with a gold fixed target. $K_{L}$ mesons travel downstream and decay into a neutral pion and two neutrinos inside the detectors. The CsI Calorimeter has 3000 channels and other detectors contributes a total of 1000 channels.

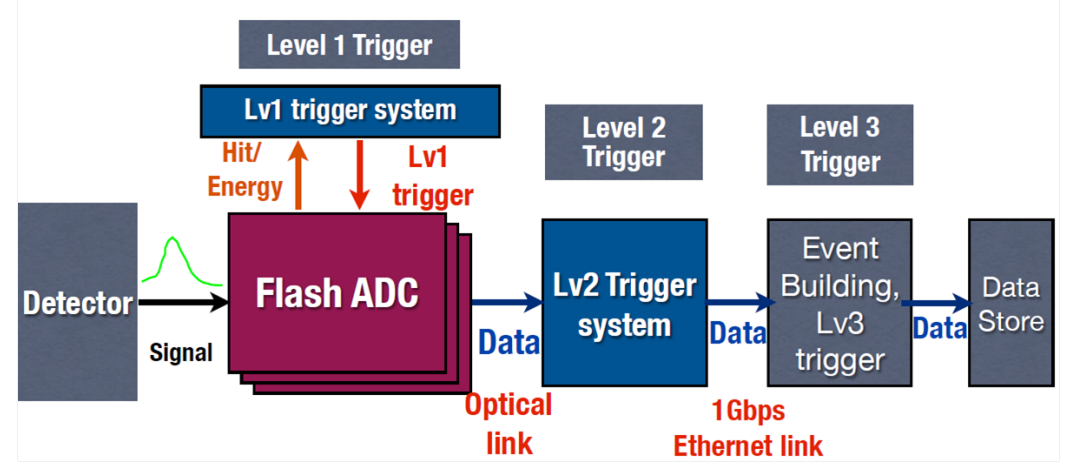

Figure 2: An overview of KOTO DAQ System.

are shaped into a Gaussian waveform of $\sim 100 \mathrm{~ns}$ at full-width at half-maximum (FWHM) using a 10-pole Bessel filter. These waveforms are then digitized at $125 \mathrm{MHz}$ and recorded for each detector channel. As indicated in Figure 3, a time-to-digital converter (TDC) is not required in our DAQ system because the sharp edge of the PMT signal can be measured via the peak of the Gaussian waveform. By fitting the Gaussian waveform with points $8 \mathrm{~ns}$ apart, we can reconstruct the timing of the PMT signals up to 1 nanosecond resolution. The timing alignment between detectors was made using beam data[8]. The data output from each ADC module is sent to the trigger system (Lv1 and Lv2) via 2.5 Gbps optical fibers. The ADC modules hold the data until the Lv1 trigger decision is made before sending the accepted events to Lv2 trigger system.

The Lv1 trigger decision is made every $8 \mathrm{~ns}$ by requiring a minimum energy in the CsI calorimeter and no activity in the veto detectors. There is a total of 16 Lv1 trigger modules in the system - each receiving information from 16 ADC modules via optical fibers. The data is summed inside each Lv1 trigger module and further summed over all Lv1 trigger modules through a daisy-chain bus. A master Lv1 trigger module receives the energy sum from the whole detector and makes a decision based on a minimum energy $(550 \mathrm{MeV})$ for the CsI calorimeter and a maximum energy for the veto detectors with varying thresholds for each detector[8].

The Lv2 trigger system retains the same hardware setup and size of the Lv1 trigger system. The Lv2 decision is made on the center of energy $(\mathrm{CoE})$ radius. The CoE radius is calculated using 

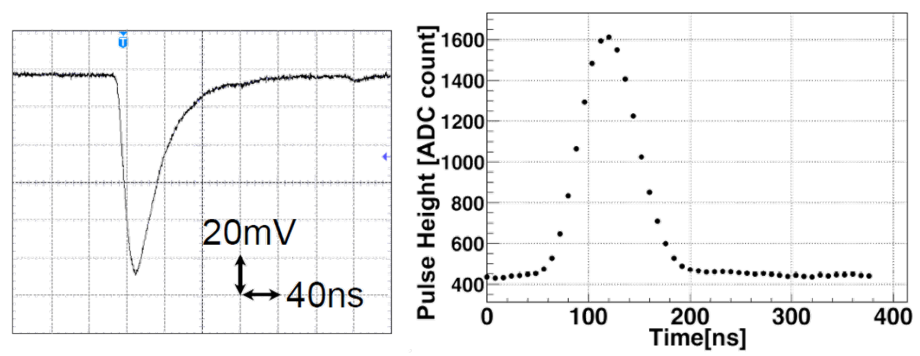

Figure 3: PMT signal from a CsI crystal recorded with an oscilloscope (left). Recorded pulse shape of the same PMT signal by the $125 \mathrm{MHz}$ ADC after the Bessel filter[8] (right).

the $\left(x_{i}, y_{i}\right)$ position of each CsI crystal weighted by its energy $E_{i}$ as:

$$
\text { CoE radius }=\frac{\sqrt{\left(\sum_{i} E_{i} x_{i}\right)^{2}+\left(\sum_{i} E_{i} y_{i}\right)^{2}}}{\sum_{i} E_{i}}
$$

Due to the missing energy and momentum from the neutrinos, we expect imbalanced energy in the transverse direction for $K_{L} \rightarrow \pi^{0} v \bar{v}$ events, and therefore large CoE radius. This allows us to distinguish our signal decay from other neutral $K_{L}$ decays like $K_{L} \rightarrow 3 \pi^{0}, K_{L} \rightarrow 2 \pi^{0}$, and $K_{L} \rightarrow 2 \gamma$. The Lv2 trigger system requires the CoE radius to be larger than $165 \mathrm{~mm}$.
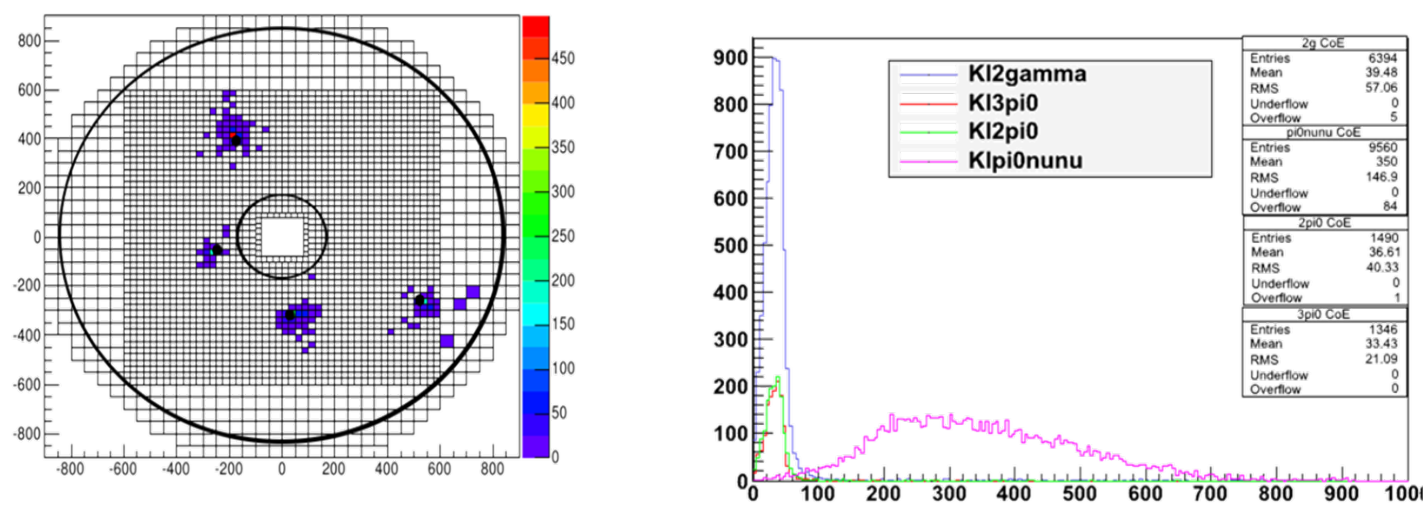

Figure 4: Left: event display of the energy deposition in the CsI calorimeter for $K_{L} \rightarrow 2 \pi^{0}$ decay; notice how the positions of the four photons clusters are symmetric along the $\mathrm{x}$ and $\mathrm{y}$ axis. Right: CoE radius distribution for different neutral $K_{L}$ decay modes generated by Monte Carlo after rejecting all the photons that fall outside the geometrical area covered by the CsI calorimeter.

The Lv3 trigger system is responsible for collecting and storing KOTO events to disk. It consists of 40 DELL nodes and each node has a 4-core processor. Each Lv2 trigger board sends out packets of event data to a network switch using UDP protocol. The switch distributes the packets to the Lv3 computer cluster. Each nodes of the Lv3 cluster runs the DAQ software with 4 threads to collect, rebuild, compress, and store events. This well matches the 4-core processor running in each node.

The first thread collects and rebuilds packets sent from different Lv2 boards via the switch. 
Each packet contains a fragment of the total event. The rebuilding process uses the $\mathrm{C}++$ map container to handle packets which may arrive out of order and store complete events in each node. The second thread runs a clustering algorithm on the rebuilt events, calculating basic information such as position and total energy for each cluster. This information can be used to perform a Lv3 cut on events. The third thread compresses event data using a bit-packing lossless algorithm. If waveform data from a channel lies within a range of $2^{n}$, then it can be packed into $n$-bits as long as the baseline data is also saved. The algorithm is optimized for minimal data movement in memory and achieves a typical compression ratio of 0.25 . The fourth thread stores event to disk in one large write to minimize the number of filesystem calls.

Typical data transfer rate from the most May, 2013 run are shown in Figure 5. All the data transfer rates are within our DAQ hardware limit. However, in order to reach the Standard Model sensitivity, we would like to investigate the limitation of our current DAQ system as the incoming event rate increases. Therefore, we used a DAQ simulation to study potential problems as reported in section 5.1 of this the paper.

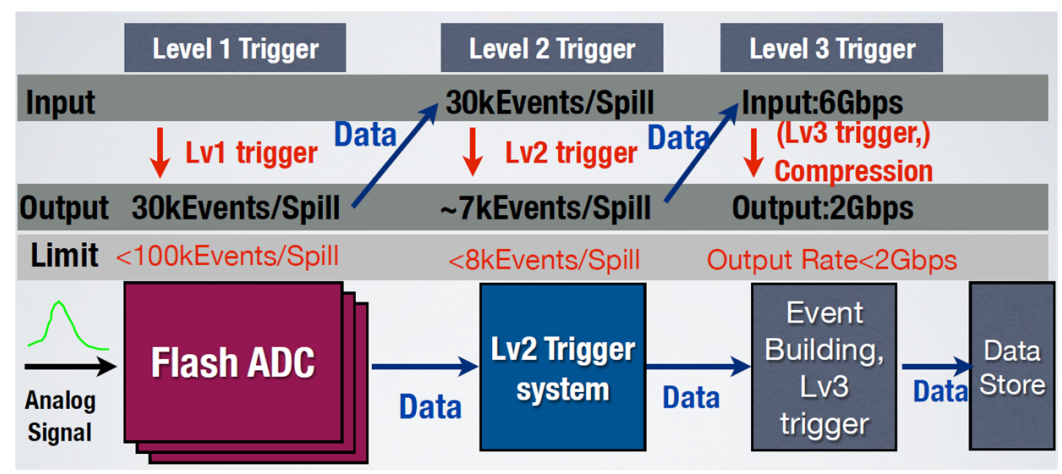

Figure 5: Current DAQ data transfer rate measured for the most recent experimental run in May, 2013.

\section{ADC Temperature Dependence}

We observed variations in ADC pedestal values while testing our cooling system during a commissioning run. A study was conducted in order to identify the relationship between the pedestal value and the temperature of the board. In order to measure the temperature of the board accurately in real time, a K-type thermocouple was attached to one of the ADC chips to arbitrarily represent the temperature of the entire board. The thermocouple was connected to an amplifier containing a cold compression reference, and wired to an Arduino microcontroller board, which read out the temperature every second.

The average pedestal and RMS for each of the 16 channel on the ADC board over a period of $\sim 13 \mu s$ was recorded at the same time as the temperature. To vary the temperature, a Variac variable transformer was used to control the voltage administered to the fans in the crate that housed the ADC board. By decreasing the voltage, the speed of the fans was slowed which caused the temperature in the crate to increase. When the temperature stabilized, a pedestal reading was taken. By taking several pedestal readings over a temperature range of about $35^{\circ} \mathrm{C}$, a clear pedestal de- 
pendence on the temperature was seen in all 16 channels corresponding to about 0.6 counts $/{ }^{\circ} \mathrm{C}$ as shown in Figure 6.

Several other studies were conducted along with the pedestal-temperature dependence, including the temperature difference across the ADC chips and the effect of the temperature on the pedestal gain and RMS values. We could not identify a single component on the board responsible for the temperature-pedestal dependence. The results of these studies were that there is a temperature gradient of about $10^{\circ} \mathrm{C}$ across the board as we move away from the fans located at the bottom of the crate. However, the temperature gradient stays the same as the temperature varied. Additionally, the gain and RMS values are not dependent upon temperature.



Figure 6: Plot of ADC pedestal values for each of the 16 channels vs. temperature: there is a clear linear dependence measured at about 0.6 counts $/{ }^{\circ} \mathrm{C}$

\section{KOTO DAQ Upgrades}

An intense $K_{L}$ flux is required to reach the standard model sensitivity to observe $K_{L} \rightarrow \pi^{0} v \bar{v}$ decay. We carried several study towards understanding the effect on our DAQ system of proton beam intensity increases in the short term $(100 \mathrm{~kW}$ ) and long term (up to $300 \mathrm{~kW}$ ). A program of upgrades was devised for the KOTO DAQ to keep the deadtime to a minimum. As the proton beam intensity increases. The short term upgrade includes online compression and a new Lv3 architecture. A long term upgrade is proposed by incorporating RCE electronics in Lv2 trigger system.

\subsection{DAQ Simulation}

The DAQ simulation models the Lv2 livetime, defined as the ratio of Lv1 events considered for Lv2 (Lv1A) and the total Lv1 requests (Lv1Req). It explores limitations of the present trigger and readout along by predicting the effect of the higher Lv1 request rate expected for increasing beam power conditions. The simulation models different parameters such as limited buffering resources, latency and bandwidth in the Lv2 board firmware and hardware as well as the rejection 
power of the CoE cut. The models predict a Lv2 livetime consistent with what was observed in the data when the parameters are set to what is avaliable in the current DAQ implementation. It also predicts that the Lv2 livetime has a much stronger dependence on the data latency and bandwidth than on buffering resources. This led to a simulation of the DAQ performance if data compression was applied online. Figure 7 shows the result of the DAQ simulation study: note the decreases of Lv2 livetime with rises of incoming event rate, and the improvements on the Lv2 livetime using data compression.

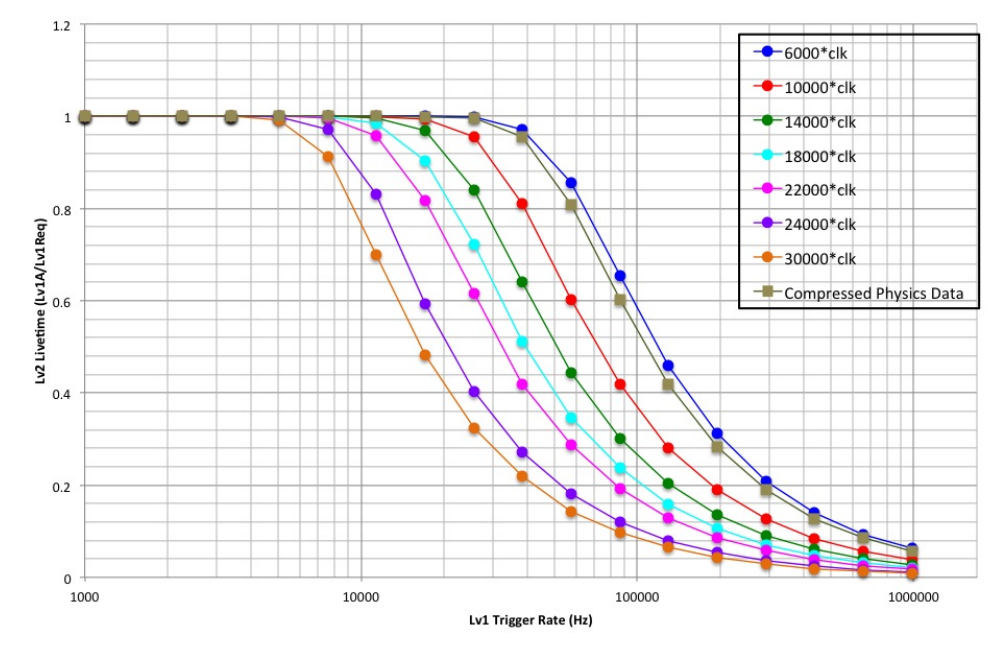

Figure 7: DAQ simulation result: the prediction for the Lv2 livetime, that is the ratio of events considered for the Lv2 decision over the total number of events of Lv1, is shown by the points for the DAQ implementation used in May, 2013. Brown squares illustrate the results if data compression is performed online.

\subsection{New Lv3 Architecture}

The current implementation of UDP protocol for the Ethernet packets flowing from Lv2 to Lv3, along with the limited computing resources of the Lv3 cluster, results in packets lost in the Lv3 trigger system. A proposed new design for the Lv3 architecture replaces the UDP protocol with Infiniband protocol for event building. Infiniband allows for remote direct memory access, memory sharing between each computer, and it uses a guaranteed delivery protocol.

In the new architecture, there will be a direct connection between each Lv2 trigger board and a Lv3 computer node. The computer nodes will share data memory and build the events via an Infiniband switch. An updated computer cluster, with 8-core single nodes, will allow to add extra threads for the Infiniband connections.

\subsection{Lv2 RCE Trigger System}

As presented in the previous section of DAQ simulation, the current DAQ system is not able to maintain a reasonable Lv2 livetime as the Lv1 trigger rate increases passed the level predicted 
for $100 \mathrm{~kW}$ beam power. The RCE project, developed by SLAC, aim at providing a set of hardware and software tools to build a customizable modern DAQ system at relatively low cost. It uses advanced telecommunication computer architecture (ATCA) for the backend communications between boards rather than daisy-chain. It is built around the Reconfigurable cluster element board, which uses a cutting-edge technology FPGA chip and native $10 \mathrm{~Gb}$ Ethernet interface for processing large amount of data with low latency. An ATCA compliant generic front module has bays for multiple RCEs and it communicates to rear transition modules which act as an interface to already exiting DAQ for different experiments. This design grants us interconnectivity and more computing power to the Lv2 trigger system so that part of the event reconstruction and filtering can be carried out before data has to be moved to Lv3 trigger system. Using the RCE boards in our Lv2 trigger system will improve the ability of our DAQ system to handle event rates up to $300 \mathrm{~kW}$ proton beam power.

\section{Conclusion}

We reported on the performance of the KOTO DAQ system is used in the May, 2013 physics run. A pipelined frontend and 3-level trigger electrics are designed to efficiently identify events with only two protons in the final state. The current DAQ system is well suited for beam power of $15 \mathrm{~kW}$ and $25 \mathrm{~kW}$ stages. The future goal is to upgrade the current DAQ system to acquire the ability to handle up to $100 \mathrm{~kW}$ and $300 \mathrm{~kW}$ beam power.

\section{Acknowledgment}

We would like to thank the conference organizers for holding TIPP' 14 and the KOTO collaborators for all of the contributions.

\section{References}

[1] J. Bord, et al. Phys. Rev. D, 83, 034030, (2011)

[2] J. K. Ahn, et al. Phys. Rev. D, 81, 072004, (2010)

[3] A. V. Artamonov, et al. Phys. Lett. 101, 191802, (2008)

[4] Y. Grossman and Y. Nir, Phys, Lett. B 398, 163 (1997)

[5] T. Masuda, Thesis, Development and Experimental Study of the KOTO Detector System Using Three $K_{L}$ Neutral Decay Modes, Kyoto University, (Preliminary)

[6] Proposal for $K_{L} \rightarrow \pi^{0} v \bar{v}$ Experiment at J-Parc, Apr. 28 (2006)

[7] M. Tecchio, et al. Physics Procedia, 37, 1940 (2012)

[8] Y. Sugiyama, et al., Data Acquisition system for the KOTO Experiment, arXiv: 1406.3907v1 (2014) 